\title{
DISRUPTIONS AND THEIR MITIGATION IN TEXTOR
}

\author{
K. H. FINKEN* Institut für Plasmaphysik, Forschungszentrum Jülich GmbH, EURATOM Association \\ Trilateral Euregio Cluster, D-52425 Jülich, Germany
}

R. JASPERS FOM-Institute for Plasma Physics Rijnhuizen, Association EURATOM-FOM Trilateral Euregio Cluster, P.O. Box 1207, NL 3430 BE Nieuwegein, The Netherlands

\author{
A. KRÄMER-FLECKEN, A. SAVTCHKOV, M. LEHNEN, and G. WAIDMANN \\ Institut für Plasmaphysik, Forschungszentrum Jülich GmbH, EURATOM Association \\ Trilateral Euregio Cluster, D-52425 Jülich, Germany
}

Received April 29, 2004

Accepted for Publication July 28, 2004

Disruptions remain a major concern for tokamak devices, particularly for large machines. The critical issues are the induced (halo) currents and the resulting forces, the excessive heating of exposed surfaces by the instantaneous power release, and the possible occurrence of highly energetic runaway electrons. The key topics of the investigations on TEXTOR in the recent years concerned (a) the power deposition pattern recorded by a fast infrared scanner, (b) the runaway generation measured by synchrotron radiation in the infrared spectral region, (c) method development for "healing" discharges that are going to disrupt, and (d) massive gas puffing for mitigating the adverse effects of disruptions.

KEYWORDS: energy quench, runaway generation, mitigation of disruptions

\section{INTRODUCTION}

Disruptions remain a major concern for tokamak devices, particularly for large machines. ${ }^{1-6}$ The critical issues are the induced (halo) currents and the resulting forces, the excessive heating of exposed surfaces by the instantaneous power release, and the possible occurrence of highly energetic runaway electrons.

It has been observed ${ }^{7,8}$ that disruptions start with internal modes; then, the confinement begins to deteriorate first in the plasma core. The plasma energy is transported from the core to the edge of the plasma, leading to an intermediate temperature rise just prior to the observation of the power quench. The proper power quench

\footnotetext{
*E-mail: k.h.finken@fz-juelich.de
}

can be a single heat pulse or a series of heat pulses. The time duration of the individual power deposition pulses is only a few ten microseconds long. Even though the instantaneous power density is extremely high, the spatial pattern of the disruptive power deposition resembles in many aspects the "normal convective" power deposition on the limiter surface..$^{9-11}$ The observations have shown that a simple diffusive process cannot adequately describe the power flux to the wall during a disruption. Ergodization, ${ }^{12,13}$ resulting from overlapping internal modes,${ }^{14-19}$ may result in a consistent description if the laminar zone, ${ }^{20,21}$ which can be understood as the open boundary of the ergodic structure, is taken into account.

In the subsequent current decay phase, the energy stored in the poloidal magnetic field is dissipated. Typical current decay time amounts to 10 to $100 \mathrm{~ms}$ and depends on the amount of released impurities during the disruption and the resulting radiation; it is rather independent of the size of the device. The fastest disruptions show a machine size dependence, and for ITER such a dependence is assumed. ${ }^{22}$ The magnetic energy of the plasma is generally higher than the thermal one. The plasma temperature in the current decay phase amounts generally to $<10 \mathrm{eV}$. This results in high plasma resistivity. Since the inductance of the electrical circuit tends to keep the plasma current constant, the loop voltage is highly increased during the current decay. This high loop voltage leads to the development of highly energetic runaway electrons (for ITER up to $100 \mathrm{MeV}$ ). When these electrons hit the vessel wall, severe damage can occur. The high loop voltage (a) induces eddy currents in conductive structures and (b) is one of the sources of halo current generation. These halo currents flow partially in the plasma and partially in structural materials. Inside the plasma, the current is forceless and follows the magnetic field lines. At locations where the current layer intersects the wall, this current is continued in the vessel; here, the current is no longer guided by the magnetic 
field and exerts very high forces to the vessel. The mitigation schemes discussed below refer to a fast cooling of the plasma and therefore predominantly affect the halo current and runaway avoidance aspects.

In the past, several topics related to disruptions were treated on TEXTOR; consequently, this paper treats the following aspects:

1. investigation of the causes for the development of disruptions

2. power release during the energy quench

3. development of runaway electrons and modeling for ITER.

In addition, special effort was undertaken to either heal disrupting plasmas or mitigate detrimental effects.

\section{INVESTIGATION OF DISRUPTION CAUSES}

The phenomenon called "disruptions" is observed in nearly all tokamak experiments. The disruption process presents a serious restriction in operating space, and it requires large technical effort to avoid disruptions. Disruptions are ordered by the path of how the stability limit is reached:

1. A strong operational limit is $q(a) \leq 2$; a low $q$-limit disruption implies the existence of the maximum permissible plasma current at a given confining magnetic field. Even though the disruption limits are normally called differently, the basic cause of disruptions at the density limit, due to strongly radiating light impurities or at-large plasma shifts as they may occur during the failure of a control system, is often the suppression of the currents at the $q=2$ surface.

2. Tearing modes are a possible source for disruptions. These are mainly connected with an unfavorable plasma current profile or high percentage of impurities and often appear in the plasma start-up or at high plasma pressures $\left(\beta_{N}\right)$.

3. The accumulation of a dense gas cloud in the vicinity of the plasma boundary, the so-called MARFE, is a potential candidate for a disruption because it leads to an unfavorable modification of the pressure and current profiles.

The performed work on plasma disruptions at TEXTOR is devoted to the tearing mode and MARFE studies. In the following both routes are described.

It has been shown ${ }^{23,24}$ that tearing modes often occur in the late current ramp-up phase. They develop at the $q=2$ surface as a small helical perturbation with a frequency of $f \sim 2 \mathrm{kHz}$. With time, the mode width increases, and its frequency decreases. Quite often, a further $m / n=1 / 1$ mode develops in the plasma center. If the islands have grown large, they can couple via $j \times B$ forces. The locking of the outer mode to the wall and the inner mode yields a huge heat pulse, which is released by the plasma and hits the inner wall components. This collapse of the plasma develops asymmetrically. The heat pulse is accompanied by an overall increase of the electron density turbulence as monitored by microwave scattering. This procedure is repeated several times until the plasma disrupts.

The MARFE instability is regularly observed in neutral beam injection (NBI) heated plasmas close to the density limit. It is a dense plasma cloud with temperatures of typically $10 \mathrm{eV}$. The MARFE appears poloidally asymmetric and moves in the poloidal direction. At the time of the MARFE formation, the density fluctuation level increases. The enhanced turbulence levels as well as the unfavorable temperature and density gradients end in a disruption.

\section{POWER RELEASE DURING THE ENERGY QUENCH}

The power released during the energy quench and deposited on the toroidal pump limiter Advanced Limiter Test-II (ALT-II) has been measured thermographically. An infrared-scanner (Inframetrix model 760) views the pair of graphite tiles of ALT-II. The infrared-scanner can operate in the "image mode" with a time resolution of $20 \mathrm{~ms}$ or at a high time resolution of $100 \mu \mathrm{s}$ in the "line scan mode," scanning only one line. The development of the surface temperature is recorded by thermography. These data are used as input data for a solver of the heat conduction equation, which returns the power flux to the surface as output. Depending on the heating problem, the partial differential equation of the heat conduction has to be solved in one, two, or three dimensions. For the given case, a two-dimensional treatment is adequate where the two directions used are the radial one and the poloidal one.

The temporal evolution of the power deposition is obtained rather straightforwardly by using the "line scan mode," as shown in Fig. 1a. The temperature, represented by false colors, changes within $100 \mathrm{~ms}$; the temperature rise is quantitatively seen in Fig. 1b. The power pulse is the sharp spike at $t=0.5 \mathrm{~s}$ and has the same width as the temperature rise, as shown in Fig. 1c; the data were obtained from the analysis by the heat equation solver. The peak heat flux amounts to $\sim 10 \mathrm{MW} / \mathrm{m}^{2}$ and is about three to four orders of magnitude higher than the average heat flux to the limiter, and the pulse occurs simultaneously with the strong spike in the $\mathrm{H}_{\alpha}$ and impurity line emissions. Our interpretation is that the heat pulse hitting wall components releases hydrogen and impurities. The amount of released impurities may have a strong influence on the subsequent dissipation rate of the poloidal magnetic field, i.e., on the current quench time.

To derive the power decay length during the disruption, knowledge of the plasma position with respect to 


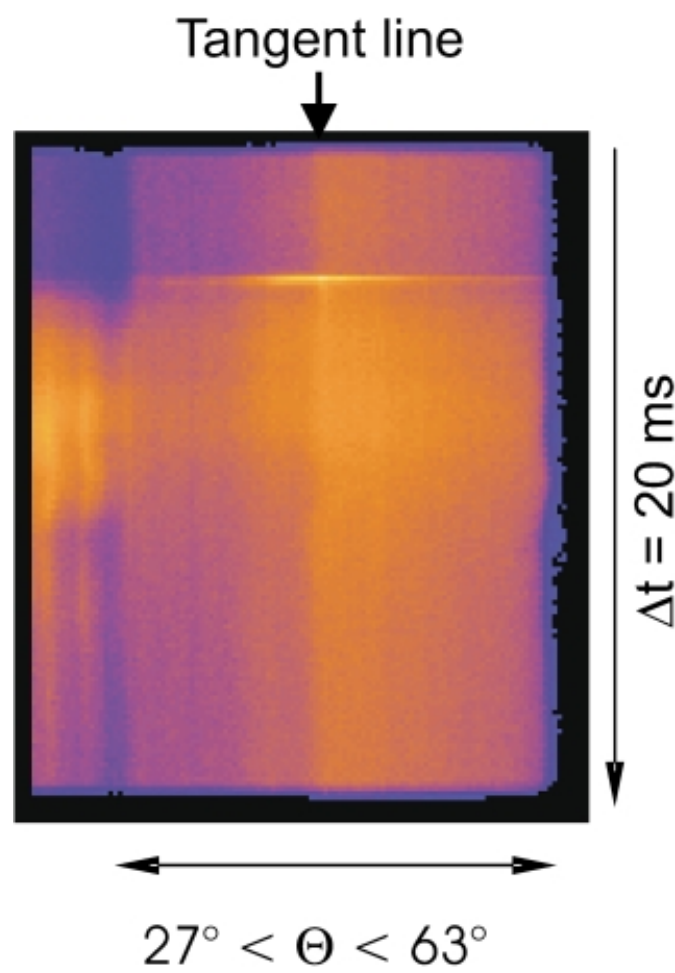

(a)
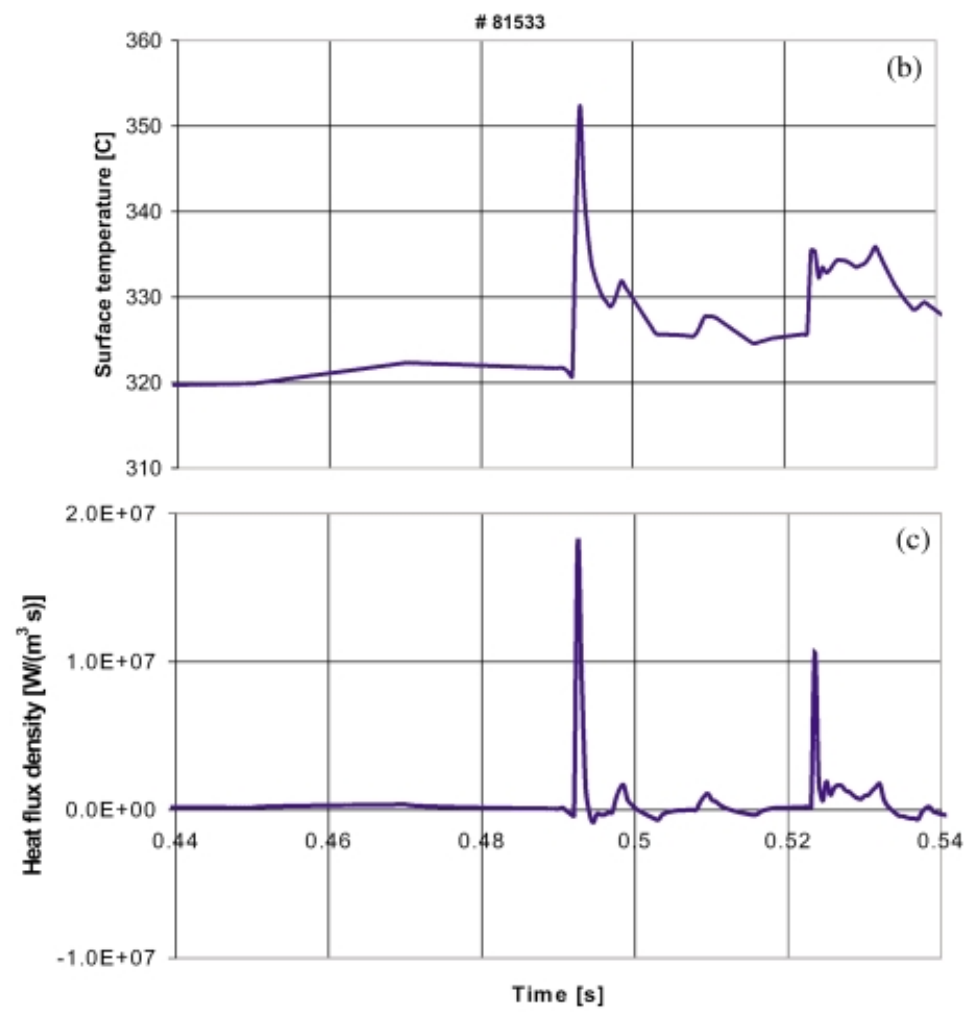

Fig. 1. (a) Thermographic picture of the surface temperature during a disruption, (b) the temperature increase, and (c) the resulting power flux density. The surface temperature of the ALT-II limiter is recorded in the line scan mode, which is a horizontal sweep over the poloidal cross section of the limiter. The time of a line gives the time resolution of $>0.1 \mathrm{~ms}$; the time of the whole picture amounts to $20 \mathrm{~ms}$. The limiter is composed of eight blades that nearly fill the belt between 38 deg at the low-field side below the equatorial midplane. The tangent line between limiter and plasma is indicated.

the limiter, the shape of the limiter surface, and the structure of the magnetic field are the essential ingredients. Since the limiter surface shows only an angle domain between 0.6 and 6 deg with respect to the magnetic field lines, all effects such as poloidal field, field ripple, etc., play a decisive role. Because of the shallow, rooflike shape of the limiter surface, only the spatial structure of those disruptions that hit the limiter centrally can be analyzed. If the plasma column hits only the top or bottom part of the limiter, it is difficult to determine the tangent

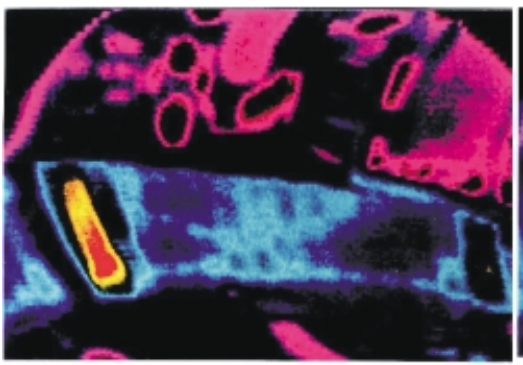

(a)

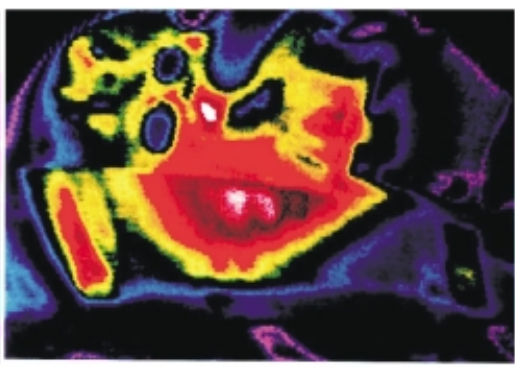

(b)

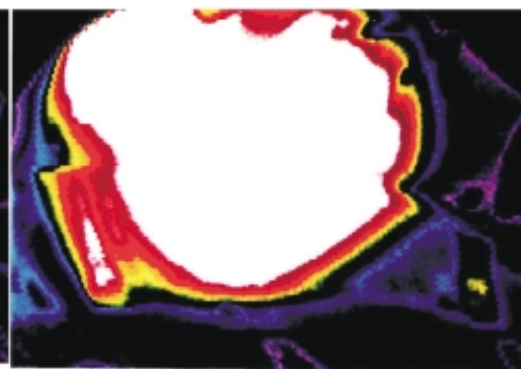

(c)

Fig. 2. View of a low-density TEXTOR-94 discharge with a thermographic camera looking in the direction of electron approach. In frame $\mathrm{A}$, recorded at $t=0.5 \mathrm{~s}$, no synchrotron radiation is observable, and only the wall structure can be recognized. In frame $\mathrm{B}$, recorded at $t=1.5 \mathrm{~s}$, the synchrotron radiation starts to develop. In frame $\mathrm{C}$, the full extent of the spot is visible from which the size of the runaway beam can be determined. 
point of the plasma and then to calculate back the incident heat pattern.

The analysis shows that the e-folding length during the disruption amounts to less than a few centimeters and is not substantially larger than the power decay length during the quiet phase of the discharge. The TEXTOR power decay length shows the additional feature that the decay length must be described by a double decay length with a value of only $\lambda_{\mathrm{P} 1}=1.5 \mathrm{~mm}$ near the tangent line of the limiter. Even the feature of the short and long decay length are reproduced during the disruption.

In the conventional model of a power decay length, a balance between a diffusive radial heat flux and a conductive/convective heat flux along the open magnetic field lines is assumed. The observed heat flux during a disruption leads here to inconsistencies: For given values of the radial temperature gradient length in the plasma and the measured heat flux, the resulting power decay length would be at least an order of magnitude larger than the one for the quiet plasma phase. This is in contrast to the infrared-based observation. We have therefore proposed a model in which ergodization plays an important role and the radial power decay is then given by the laminar zone of the open magnetic field lines of the ergodized system. ${ }^{13}$ The precursor modes play the role of generators obtaining sufficient plasma ergodization.

\section{RUNAWAY ELECTRONS}

The phenomenon of electron runaway is an interesting and well-known result of the fact that the mean free path of an electron in the plasma is a strongly decreasing function of its velocity. In an electric field, electrons that exceed a critical velocity for which the collisional drag balances the acceleration by the field are accelerated freely and can reach very high energies. Runaway electrons have been observed in nearly all tokamaks, mainly during disruptions (due to the high induced electric field), where they can reach energies of several tens of megaelectron-volts (Refs. 25, 26, and 27). They are also encountered in other fields of plasma physics, e.g., in solar flares or thunderstorms. ${ }^{28}$ It is of major importance to know the processes of runaway generation and runaway loss during disruptions because of the severe damage the local loss of large numbers of these highly energetic electrons may cause on first-wall components. ${ }^{29}$

Over the last decade, runaway studies have been performed at TEXTOR. These experiments were unique in the fact that the synchrotron radiation of relativistic electrons in the energy range of 20 to $30 \mathrm{MeV}$ was exploited to observe confined runaway electrons. These measurements yielded a wealth of information on runaway electron behavior, the highlights of which are reviewed here.

\section{IV.A. Synchrotron Radiation}

Synchrotron radiation is a powerful tool for diagnosing the relativistic runaway electron distribution. This diagnostic provides a direct image of the runaway beam inside the plasma, recorded with a thermographic camera. From the spectral features the runaway energy can be obtained, the intensity of the radiation is a measure of the number of runaway electrons, and the synchrotron spot carries information on their perpendicular momentum and spatial distribution. Figure 2 gives an example of such synchrotron measurement at TEXTOR-94 in the wavelength range of 3 to $8 \mu \mathrm{m}$ where the emission reaches its maximum. Detailed information on the synchrotron emission by relativistic electrons and the diagnostic can be found in Ref. 30. The required electron energy for the generation of synchrotron radiation amounts to $W_{R} \sim 25 \mathrm{MeV}$, which requires a free-fall time of $\sim 1 \mathrm{~s}$ at a loop voltage of $1 \mathrm{~V}$. The typical runaway current in "normal" low-density discharges $\left(n_{e}<1 \times 10^{19} \mathrm{~m}^{-3}\right)$ in TEXTOR is 5 to $10 \mathrm{kA}$ at a plasma current of typically $350 \mathrm{kA}$.

\section{IV.B. Runaway Generation}

Two mechanisms for the generation of runaway electrons are described by theory. The first, which we refer to as primary generation, treats the diffusion in the velocity space of the electron distribution around the critical velocity. The production rate in this process depends exponentially on the parameter $\varepsilon=E / E_{\text {crit }}$, where $E$ is the electric field and $E_{c r i t}$ is the field for which a thermal electron would run away: $E_{\text {crit }} \sim Z_{\text {eff }} n_{e} / T_{e}$. The second mechanism, secondary generation, is the process in which already existing highly energetic electrons kick thermal electrons into the runaway regime by close Coulomb collisions. The production rate in this case is proportional to the number of runaway electrons and is therefore expected to lead to an exponential growth rate of the runaway generation. ${ }^{31}$ Because of this it is often referred to as the avalanche mechanism. Another important difference between the primary and secondary process is the very weak density dependence of the latter. The increased critical velocity at higher densities, which makes it more difficult for secondary electrons to reach the runaway region, is compensated by the increased number of collisions. In a simplified treatment the secondary process can be parameterized by the avalanche time $t_{0}$, the time in which existing runaway electrons produce new runaway electrons:

$$
t_{0}(\mathrm{~s}) \approx 0.015 \cdot\left(2+Z_{\text {eff }}\right) / E(\mathrm{~V} / \mathrm{m}) .
$$

If the runaway confinement time is longer than $t_{0}$, an exponentially increasing runaway population will be observed.

Predictions of runaway production during disruptions in future tokamak reactors are crucially dependent 
on the assumption of whether or not the secondary generation dominates. The first experimental evidence for this process has been reported from TEXTOR (Ref. 32). This is illustrated most convincingly in Fig. 3; the discharge is a "normal" low-density discharge without a disruption as we have been using to study runaway electron properties. Analysis of the time behavior of the synchrotron radiation at low-density $\left[n_{e}(0) \approx 1 \times 10^{19} \mathrm{~m}^{-3}\right]$ discharges showed the exponential increase and the weak density dependence expected for the avalanche mechanism. Note that for the case under consideration, the synchrotron radiation signal is almost directly proportional to the number of confined runaway electrons.

\section{IV.C. Disruptions}

In a few instances the generation of runaway electrons during major disruptions has been observed with the synchrotron radiation diagnostic on TEXTOR. An example ${ }^{33}$ shows that there is a delay of a few milliseconds between the thermal quench and the observation of nonthermal electron cyclotron emission (ECE) and a small beam of highly energetic runaway electrons as witnessed by the synchrotron radiation. Probably, in the phase in between, the magnetic configuration was still stochastic, preventing runaway electrons to be confined long enough to reach high energies.

Another observation made was that once the synchrotron radiation was observed, the intensity did not increase further, irrespective of the high loop voltage measured. This indicates that in the center of the plasma, the runaway current was nearly as large as the plasma

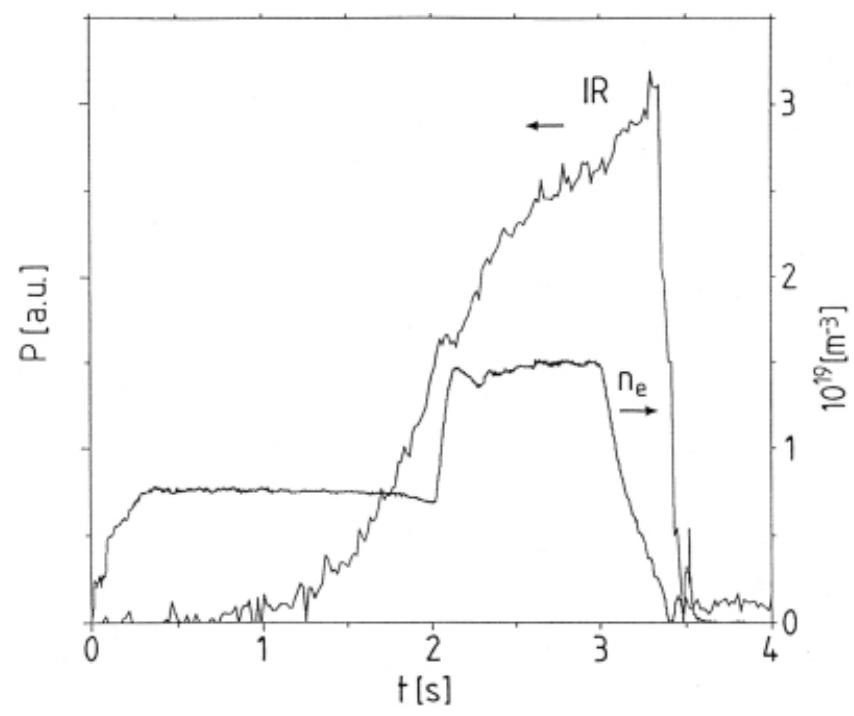

Fig. 3. Evidence for secondary generation of runaway electrons on TEXTOR. Time trace of the infrared synchrotron radiation shows an exponentially increase and continues to increase when the density is doubled. current before the disruption. Based on these observations, a simple zero-dimensional model was developed that described the TEXTOR data reasonably well by taking into account the secondary generation mechanism. One prediction of this study is that in a reactor, a high runaway generation rate during a disruption will prevent the runaway electrons from reaching high energies and so limits the damage, compared to a modest production rate. Typical values for ITER are a runaway current of more than half of the plasma current, maximum runaway energy of up to $50 \mathrm{MeV}$, and total energy in the runaway beam of $\sim 100$ MJ. Similar predictions are obtained from a more sophisticated modeling, using a kinetic code (ARENA) and treating the primary and secondary generation as well as pitch angle scattering, momentum diffusion, and radiation damping. ${ }^{34}$ Nevertheless, a complete suppression of the runaway generation will be favored, and attempts to achieve this are described in Sec. VI.

\section{DETECTION AND HEALING OF DISRUPTIONS}

Most TEXTOR disruptions show in the beginning an $m / n=2 / 1$ magnetohydrodynamic activity in the gradient region at $r / a \sim 0.6$. The duration of this phase of so-called disruption precursors varies strongly with the temporal development of plasma parameters as electron density and impurity content. The common feature is an increase of the mode width together with a decrease of the rotation frequency (Sec. II). The detection of this precursor offers a method for (a) healing of the disruption with appropriate means as heating of the island or injection of toroidal momentum or (b) a controlled rampdown of the plasma current or a mitigation of the disruption by a massive gas puff (Sec. VI). A new method at TEXTOR (Refs. 35 and 36) for the detection of the $m / n=$ $2 / 1$ island is based on specific properties of the mode. The $m / n=2 / 1$ mode is a helical perturbation of the plasma current, which consists of two poloidal windings for one toroidal circulation. The simultaneous observation of the oscillation on the low- and high-field side of the $q=2$ surface is used for the detection of the mode. A dynamic cross correlation of the two radial separated observations yields a cross-correlation coefficient. If the cross-correlation coefficient exceeds a preselected level, a trigger is generated and used to heal or mitigate the imminent disruption.

Because of the current perturbation, the electron density, temperature, and magnetic field are perturbed. The electron temperature diagnostic (ECE) at TEXTOR is well localized $(\Delta r \sim 0.01 \mathrm{~m})$ to measure the temperature oscillations of the mode. The time for the detection of the mode depends on the amplitude of the mode and its position with respect to the observation volume of the ECE diagnostic and varies between 1.5 and $25 \mathrm{~ms}$. The trigger onset is controlled by the threshold set for 


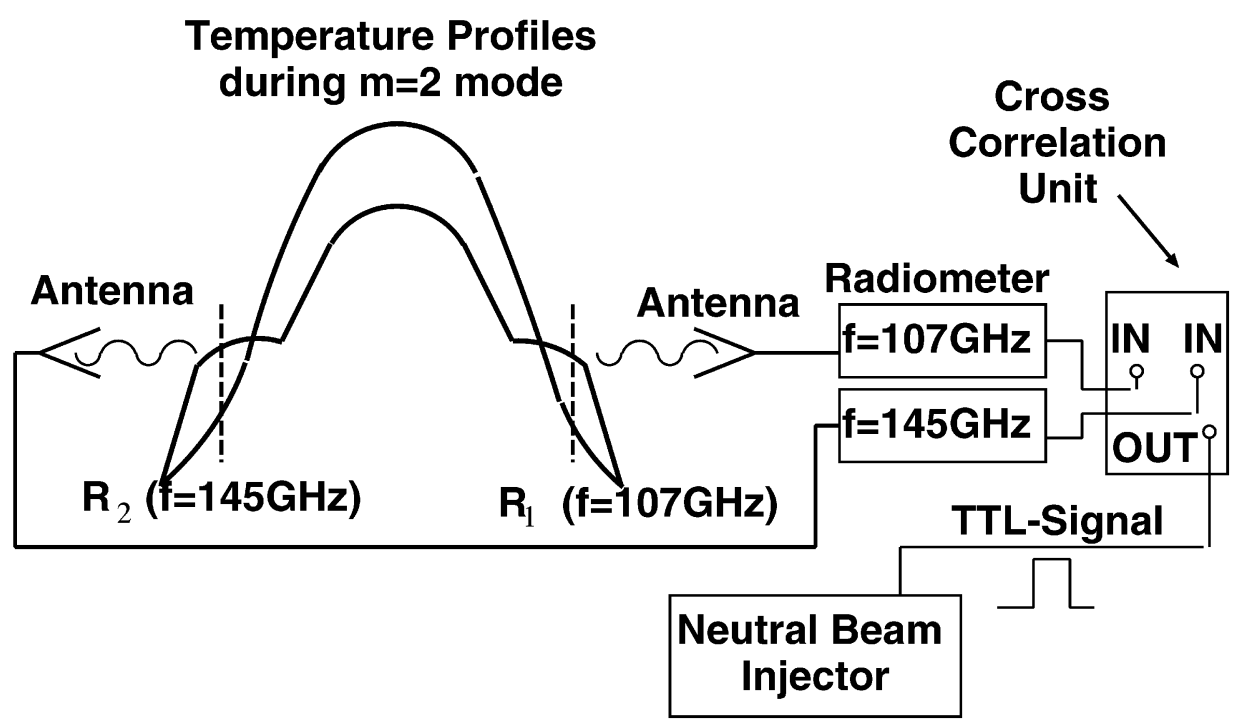

Fig. 4. The precursor detection with two ECE channels as implemented at TEXTOR.

the cross-correlation coefficient. At a low trigger setting, the reaction time for healing is sufficiently long, but the reaction may be premature. The high trigger level moves its onset closer to the disruption and makes it more difficult to heal a disruption. The setup at TEXTOR is shown in Fig. 4. The $q=2$ surface is observed by two radiometers measuring the electron temperature. The plasma profiles are shown for the two extreme cases,

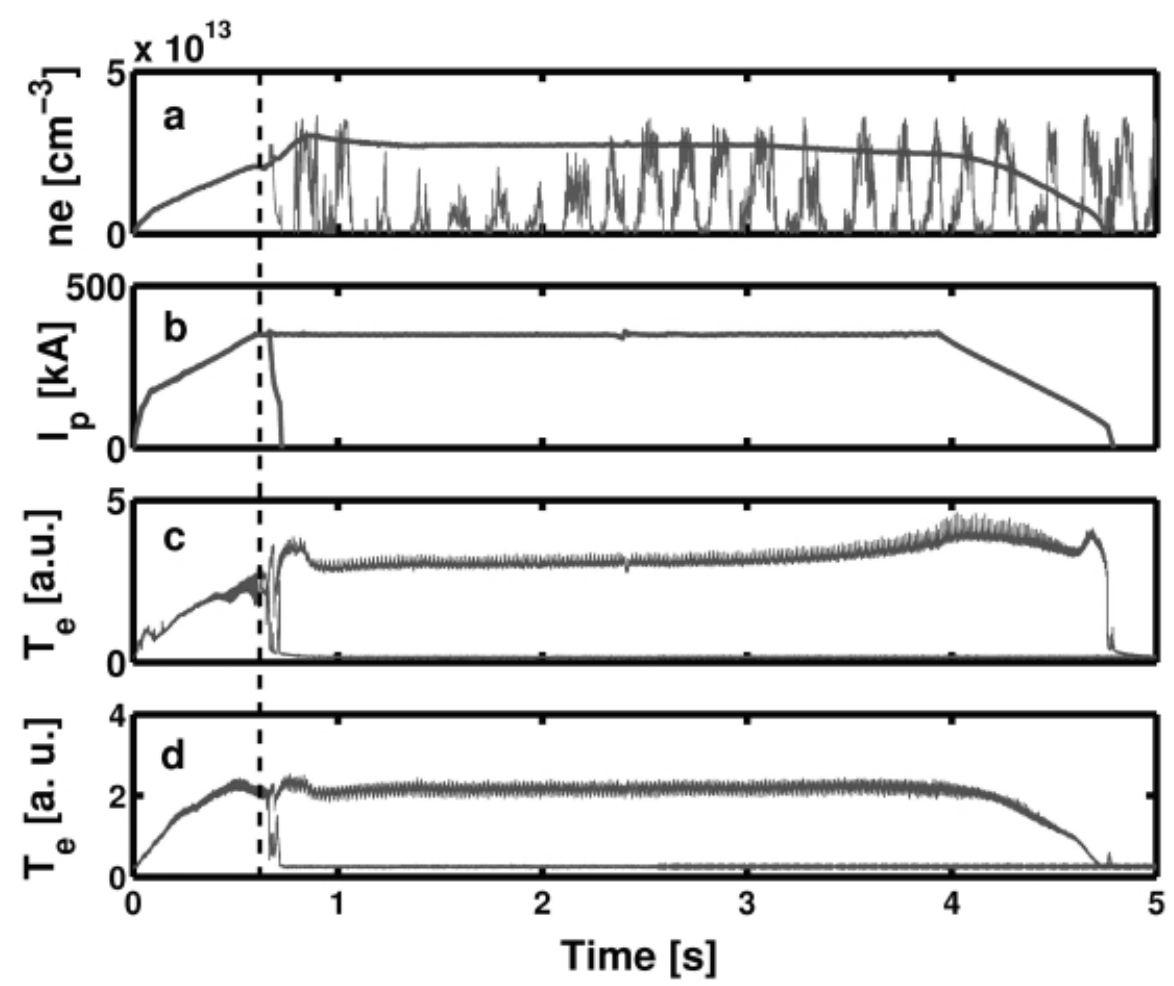

Fig. 5. Comparison of two discharges with [\#88264 (solid line)] and without [\#88260 (dashed line)] NBI. (a) Line-averaged density, (b) plasma current, (c) ECE—channel at $R=2.1 \mathrm{~m}$, (d) central ECE—channel. The dashed vertical line marks the onset of the tangential NBI. 
$\mathrm{X}$ - and O-points of the island. For disruption healing, the trigger starts the tangential co-current NBI.

The tangential NBI is used to add additional toroidal momentum in the plasma and the island, respectively. This will generate a velocity shear across the island, and as a consequence the mode is torn apart. An example of the method is shown in Fig. 5. Two discharges are shown with the same plasma parameters as seen with the evolution of plasma current and density. Both discharges show an early mode activity as seen with the time traces of the ECE channel at the $q=2$ surface. Discharge \#88260 (dashed line) disrupts. For discharge \#88264 (solid line), the tangential neutral beam was switched on. It prevents the disruption by generating a velocity shear across the island. After a minor disruption that is visible by the stationary sawtoothing in the plasma center, the plasma recovers.

\section{MITIGATION OF DETRIMENTAL EFFECTS CAUSED BY DISRUPTIONS}

It was discussed in Sec. I that the detrimental effects of disruptions are the excessive heat flux due to the thermal energy quench and due to the dissipation of the magnetic energy in the current decay phase, the forces due to halo currents, and the effects of the runaway electrons hitting vessel components. The warning time for disruptions can amount to some hundred milliseconds in favorable cases, e.g., density limit disruptions, it can, however, also be as short as $\sim 10 \mathrm{~ms}$ as, e.g., for vertical displacement events in elongated plasmas. In order to mitigate a disruption, one needs a very fast reacting system that provides a fast and massive gas injection. "Massive" means with respect to the particle content of the discharge or such amount of gas that a large fraction of the energy content (thermal and magnetic) can be radiated. Under discussion are "killer pellets," fast liquid jets, and heavy gas injection.

For the investigations on TEXTOR, we have concentrated on heavy gas injection ${ }^{37}$ particularly with the aim to avoid or stop runaway electrons. For this reason a fast gas injection valve that releases 5 to $250 \mathrm{ml}$ of gas at a pressure of 0 to 30 bars into the discharge vessel has been developed. The valve is opened by an eddy current acting on an aluminum stem and can be located in the full toroidal field very close to the discharge. This minimizes the delay due to the gas stream, which propagates at sound velocity. The valve is open within $0.5 \mathrm{~ms}$ after a trigger signal.

On TEXTOR we have used helium gas for the injection. Helium has successfully been used before to stop energetic runaway electrons in a low-density runaway discharge. This injection of helium reduces the synchrotron radiation from the plasma and the $\mathrm{X}$-ray and neutron emission at the end of the discharge by more than an order of magnitude. Helium is not implanted in the walls, and the start of the following discharge is not disturbed by the presence of strongly radiating impurities. The stopping of the runaway electrons has been treated in Ref. 38 as the balance of (a) the accelerating forces by the loop voltage, (b) the momentum loss due to the synchrotron radiation, and (c) the drag force due to the collisions. For the TEXTOR case, a slowing-down time of $1 \mathrm{~s}$ has been estimated well in agreement with the experimental data.

Since the TEXTOR device is too small to generate a detectable amount of runaway electrons with sufficient high energy for the emission of synchrotron radiation, we have tested the concept of runaway stopping ${ }^{39}$ in a lowdensity discharge. Figure 6 shows an image of the infrared scanner of such a runaway discharge containing the synchrotron radiation as the bright object from the top of the image to the middle. Shortly before the time marked by the top arrow, the fast valve is triggered. One sees that the runaway electrons start to react within $0.5 \mathrm{~ms}$. As in the steady-state conditions, the runaways are quickly slowed down by the helium and are not hitting the wall. The proper disruption due to the massive helium influx occurs $\sim 2 \mathrm{~ms}$ after the trigger signal.

\section{Loss of runaway electrons after fast He puff}

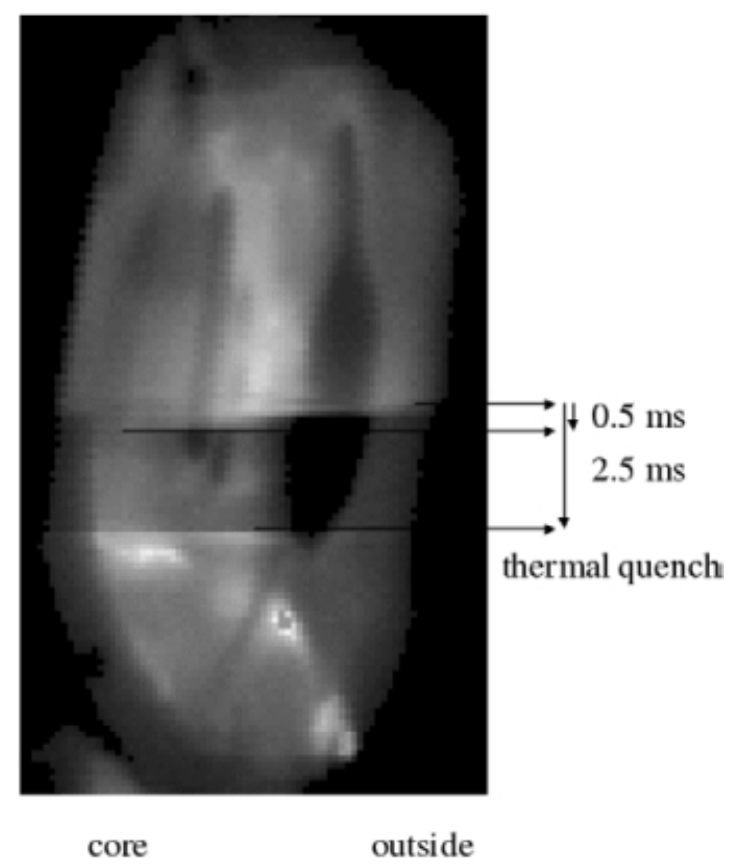

Fig. 6. Loss of runaway electrons and thermal heat pulse in infrared picture. The image contains both the local and temporal information because two sweeping mirrorsone horizontal and one vertical—create the image. The time proceeds from top to bottom and amounts to $20 \mathrm{~ms}$ for a full frame. The top part of the figure is dominated by synchrotron radiation from steady-state runaways; at the time of the top arrow, the fast gas valve is opened, and within $0.5 \mathrm{~ms}$ the runaway electrons disappear. The induced disruption and its associated thermal quench occur 2 ms later. 


\section{SUMMARY}

TEXTOR has long-term experience on investigations of disruptions. The topics cover many aspects, starting with observations of the precursors and then the problem of the power deposition, the generation of highenergy runaway electrons, the proposal of healing a discharge that is going to disrupt, and finally methods to mitigate detrimental effects of disruptions. Since the topic is relevant for ITER, it will be investigated further.

\section{REFERENCES}

1. O. GRUBER et al., Plasma Phys. Control. Fusion, 35, B191 (1993).

2. G. PAUTASSO et al., Nucl. Fusion, 34, 455 (1994) .

3. J. A. WESSON et al., Nucl. Fusion, 29, 641 (1989).

4. J. A. WESSON et al., Nucl. Fusion, 30, 1011 (1990).

5. D. J. CAMPBELL et al., Nucl. Fusion Supplement, 1(IAEA-CN47), 433 (1987).

6. F. C. SCHUELLER, Plasma Phys. Control. Fusion, 37, A135 (1995).

7. K. H. FINKEN et al., Nucl. Fusion, 32, 915 (1992).

8. K. H. FINKEN, T. DENNER, and G. MANK, Nucl. Fusion, 40 , 339 (2000).

9. T. DENNER, K.H. FINKEN, G. MANK, and N. NODA, Nucl. Fusion, 39, 83 (1999).

10. K. H. FINKEN et al., J. Nucl. Mater., 162-164, 655 (1989).

11. K. H. FINKEN et al., J. Nucl. Mater., 196-198, 220 (1992).

12. A. B. RECHESTER and M. N. ROSENBLUTH, Phys. Rev. Lett., 40, 38 (1978).

13. K. H. FINKEN, A. KRAEMER-FLECKEN, G. MANK, and S. S. ABDULLAEV, J. Nucl. Mater., 290-293, 1064 (2001).

14. A. BONDESON et al., Nucl. Fusion, 31, 1695 (1991).

15. G. Z. A. HUYSMANS et al., Control. Fusion Plasma Phys., 19C, I, 201 (1995).
16. P. SMEULDERS et al., Control. Fusion Plasma Phys., 19C, IV, 61 (1995).

17. F. TROYON et al., Plasma Phys. Control. Fusion, 26, 209 (1984).

18. R. B. WHITE et al., Phys. Fluids, 20, 800 (1977).

19. S. MAZUR, Phys. Plasmas, 1, 3356 (1994).

20. PH. GHENDRIH, A. GROSMAN, and H. CAPES, Plasma Phys. Control. Fusion, 38, 1653 (1996).

21. K. H. FINKEN, T. EICH, and A. KALECK, Nucl. Fusion, 38, 515 (1998).

22. ITER PHYSICS EXPERT GROUP, Nucl. Fusion, 39, pp. 2251 and 2333 (1999).

23. G. WAIDMANN, in Contributions to High Temperature Plasma Physics, p. 457, Akademie Verlag (1994).

24. G.WAIDMANN and G. KUANG, Nucl. Fusion, 32, 645 (1992).

25. H. KNOEPFEL and D. A. SPONG, Nucl. Fusion, 19, 785 (1979).

26. R. D. GILL et al., Nucl. Fusion, 40, 163 (2000).

27. K. H. FINKEN et al., Nucl Fusion, 30, 859 (1990).

28. A. V. GUREVICH et al., Radio Science, 31, 1541 (1996).

29. S. PUTVINSKI et al., Plasma Phys. Control. Fusion, 39, B157 (1997).

30. R. JASPERS et al., Rev. Sci. Instrum., 72, 466 (2001).

31. M. N. ROSENBLUTH and S. V. PUTVINSKI, Nucl. Fusion, 37, 1355 (1997); see also YU. A. SOLOKOV, JETP Lett., 29, 218 (1979).

32. R. JASPERS et al., Nucl Fusion, 33, 1775 (1993).

33. R. JASPERS et al., Nucl. Fusion, 36, 367 (1996).

34. P. HELANDER, L. G. ERIKSON, and F. ANDERSON, Plasma Phys. Control. Fusion, 44, B247 (2002).

35. A. KRAEMER-FLECKEN et al., Fusion Eng. Des., 56-57, 773 (2002).

36. A. KRAEMER-FLECKEN et al., Nucl Fusion, 43, 1437 (2003).

37. A. SAVTCHKOV, K. H. FINKEN, and G. MANK, Rev. Sci. Instrum., 73, 3490 (2002).

38. K. H. FINKEN, G. MANK, A. KRAEMER-FLECKEN, and R. JASPERS, Nucl. Fusion, 41, 1651 (2001).

39. K. H. FINKEN, A. KRAEMER-FLECKEN, M. LEHNEN, and A. SAVTCHKOV, J. Nucl. Mater., 313-316, 1247 (2003). 